\title{
Effects of cryodevice type and donors' sexual maturity on vitrification of minke whale (Balaenoptera bonaerensis) oocytes at germinal vesicle stage
}

\author{
Hiroshi Iwayama ${ }^{1}$, Shinichi Hochi ${ }^{2}$, Megumi Kato ${ }^{3}$, Masumi Hirabayashi ${ }^{3}$, Masashige Kuwayama ${ }^{4}$, \\ Hajime Ishikawa ${ }^{5}$, Seiji Ohsumi $i^{5}$ and Yutaka Fukui ${ }^{1}$ \\ Obihiro University of Agriculture and Veterinary Medicine, Shinshu University, National Institute for Physiological Sciences, \\ Okazaki, Kato Ladies Clinic, Tokyo and The Institute of Cetacean Research, Tokyo, Japan
}

Date submitted: 13.07.04. Date accepted: 21.09.04

\section{Summary}

Germinal-vesicle-stage oocytes enclosed with compact cumulus cell layers (COCs) were recovered from adult or prepubertal minke whale ovaries, and were vitrified in a solution containing $15 \%$ ethylene glycol, $15 \%$ DMSO and $0.5 \mathrm{M}$ sucrose using either a Cryotop or an open-pulled straw (OPS) as the cryodevice. The post-warm COCs with normal morphology were cultured for $40 \mathrm{~h}$ in a 390 mosmol in vitro maturation medium, and oocytes extruding the first polar body were considered to be matured. The proportion of morphologically normal COCs after vitrification and warming was higher when the COCs were cryopreserved by Cryotop (adult origin, $88.4 \%$; prepubertal origin, $80.8 \%$ ) compared with the OPS (adult origin, 67.7\%; prepubertal origin, 64.2\%). The oocyte maturation rate was higher in the adult/Cryotop group (29.1\%) compared with those of the prepubertal/Cryotop group (14.4\%), the adult/OPS group (14.3\%) and the prepubertal/OPS group (10.6\%). These results indicate that the Cryotop is a better device than the OPS for vitrification of immature oocytes from adult minke whales.

Keywords: Cryotop, Immature oocytes, Minke whale, OPS, Sexual maturity

\section{Introduction}

A limited number of reports are available to understand the fundamental aspects of gamete interaction regarding fertilization in marine mammals. The nuclear maturation rate of minke whale oocytes is approximately 30\%, even after $120 \mathrm{~h}$ of in vitro maturation (IVM) culture (Fukui et al., 1997a; Asada

All correspondence to: Yutaka Fukui, Laboratory of Animal Reproduction, Obihiro University of Agriculture and Veterinary Medicine, Obihiro, Hokkaido 080-8555, Japan. Tel: +81155495415. Fax: +81 155495593 . e-mail: fukui@obihiro.ac.jp

${ }^{1}$ Laboratory of Animal Reproduction, Obihiro University of Agriculture and Veterinary Medicine, Obihiro, Hokkaido 080-8555, Japan.

${ }^{2}$ Faculty of Textile Science and Technology, Shinshu University, Ueda, Nagano 386-8567, Japan.

${ }^{3}$ National Institute for Physiological Sciences, Okazaki, Aichi 444-8787, Japan.

${ }^{4}$ Kato Ladies Clinic, Tokyo 160-0023, Japan.

${ }^{5}$ The Institute of Cetacean Research, Tokyo 104-0055, Japan. et al., 2001a). The cleavage rate of the IVM oocytes after in vitro fertilization (IVF) and intracytoplasmic sperm injection (ICSI) are also low, at approximately $15 \%$ and $5 \%$, respectively (Fukui et al., 1997b; Asada et al., 2001b). A practical cryopreservation technique for immature whale oocytes has been desired, because one of the factors restricting research into the reproductive physiology of whales is the difficulty of carrying culture devices $\left(\mathrm{CO}_{2}\right.$ incubator and cylinder) onto a research base ship. However, conventional two-step freezing of immature minke whale oocytes has resulted in limited success (Asada et al., 2000).

Vitrification protocols characterized by an ultrarapid cooling rate have been shown to be promising methods for oocyte cryopreservation. Newly developed cryodevices for accelerating the cooling rate include electron microscope grids (Martino et al., 1996), the open-pulled straw (OPS; Vajta et al., 1998), the Cryoloop (Lane et al., 1999) and the Cryotop (Kuwayama \& Kato, 2000). Minor modifications of the container in the OPS system have also been reported (Kong et al., 2000; Hochi et al., 2001; Tominaga \& 
Hamada, 2001). Hochi et al. (2004) reported that the Cryotop is a better device than the OPS or Cryoloop for vitrification of 1-cell-stage rabbit zygotes.

The present study was undertaken as a part of the Japanese Whale Research Program with Special Permit in the Antarctic Sea (the research area: $60^{\circ} \mathrm{S}$ to the ice edge and $130^{\circ} \mathrm{E}$ to $170^{\circ} \mathrm{W}, 60^{\circ}$ to $69^{\circ} \mathrm{S}$ and $140^{\circ}$ to $\left.170^{\circ} \mathrm{W}\right)$. Sampling from minke whales was conducted from 2 December 2002 to 8 March 2003 by the Institute of Cetacean Research, Tokyo, Japan. The effects of different cryodevices (Cryotop vs OPS) and levels of sexual maturity of the oocyte donors (prepubertal vs adult) on the vitrification of germinal vesicle (GV)-stage oocytes were examined. Oocyte survival was evaluated by morphology immediately after warming and by nuclear progression to the second metaphase during IVM culture.

\section{Materials and methods}

The present study was approved by the Animal Experimental Committee of Obihiro University of Agriculture and Veterinary Medicine, in accordance with the Guiding Principles for the Care and Use of Research Animals.

\section{Animals}

All whales used in the present study were killed by explosive harpoons, which have been recognized as the most humane killing method for whales by the International Whaling Commission (IWC) and are included in Schedule III (Capture) in the International Convention for the Regulations of Whaling. Special attention to reduce the time to death was given to all sampled whales; explosive harpoons were used for the primary method, and a large-caliber rifle for the secondary method when necessary.

The sexual maturity of minke whales was determined by the presence of corpus luteum and/or corpora albicans on either ovary, and animals with neither corpus luteum nor corpora albicans were considered prepubertal. A total of 88 female minke whales (prepubertal: $n=32,6.5 \pm 0.2 \mathrm{~m}$ and $3.4 \pm 0.2 \mathrm{t}$; adult: $n=56,8.8 \pm 0.6 \mathrm{~m}, 7.9 \pm 0.2 \mathrm{t}$, for mean body length and body weight, respectively) were available as ovary donors. All adult whales were found pregnant by the presence of a fetus in their uteri. Ovaries were collected within $3 \mathrm{~h}$ after the death of donor whales.

\section{Oocyte collection}

Cumulus-oocyte complexes (COCs) were collected by aspirating follicles $2-15 \mathrm{~mm}$ in diameter with a $20 \mathrm{ml}$ syringe and 18-gauge needle. The COCs were classified into four groups according to the ooplasm and cumulus morphology. Only oocytes surrounded by more than two layers of cumulus cells and granulated evenly were selected for vitrification (Fig. 1A). The COCs were washed with Medium 199 (Sigma, St Louis, $\mathrm{MO})$ containing $0.1 \%(\mathrm{w} / \mathrm{v})$ polyvinylalcohol, $2 \mathrm{mM}$ $\mathrm{NaHCO}_{3}, 10 \mathrm{mM}$ HEPES and $75 \mu \mathrm{g} / \mathrm{ml}$ kanamycin, hereafter referred to as Hepes-M199.

\section{Vitrification of COCs}

Equilibration of cryoprotective agents to the COCs was performed at ambient temperature $\left(20^{\circ} \mathrm{C}\right)$. The COCs were placed in Medium 199 supplemented with $20 \%(\mathrm{v} / \mathrm{v})$ calf serum (M199/CS). The COCs were first exposed to $7.5 \%$ ethylene glycol (EG) in M199/CS for $8 \mathrm{~min}$, and then 7.5\% EG and 7.5\% DMSO in M199/CS for $4 \mathrm{~min}$. Finally, the COCs were transferred into 15\% EG, 15\% DMSO and 0.5 M sucrose in M199/CS. Within $1 \mathrm{~min}, 5-10$ COCs were placed on a sheet of a Cryotop (Kitazato Supply Co., Tokyo, Japan) or at the end of an OPS with a minimum volume of the vitrification solution, and were plunged into liquid nitrogen. After storage in liquid nitrogen and transportation to a laboratory, the COCs were warmed by immersing the Cryotop or OPS in 0.5 M sucrose in M199/CS for 5 min, and then transferred to M199/CS for $5 \mathrm{~min}$ at $37^{\circ} \mathrm{C}$.

\section{In vitro maturation}

Based on preliminary measurement of the osmolarity of whale follicular fluid (wFF: $n=23,387.9 \pm 3.1$ mosmol), osmolarity of the IVM medium was adjusted to 390 mosmol by changing the concentration of $\mathrm{NaCl}$, $\mathrm{KCl}, \mathrm{MgSO}_{4}$ (anhydrous) and $\mathrm{CaCl}_{2} \cdot 2 \mathrm{H}_{2} \mathrm{O}$ at a constant ratio with M199. Supplements to the M199 were $10 \%$ (v/v) wFF, $0.33 \mathrm{mM}$ sodium pyruvate (Wako Pure Chemical Industries, Osaka, Japan), $1 \mathrm{mM}$ glutamine (Wako), $10 \mathrm{ng} / \mathrm{ml}$ epidermal growth factor (Sigma), $100 \mu \mathrm{M}$ cysteamine (Sigma), $75 \mu \mathrm{g} / \mathrm{ml}$ kanamycin, $1 \mu \mathrm{g} / \mathrm{ml}$ estradiol-17 $\beta$ (Sigma) and $0.02 \mathrm{AU} / \mathrm{ml} \mathrm{pFSH}$ (Antrin; Kawasaki Pharmaceutical Co., Kanagawa, Japan). The COCs were cultured in $50 \mu \mathrm{l}$ droplets of the IVM medium covered with mineral oil for $40 \mathrm{~h}$ at $37^{\circ} \mathrm{C}$ in $5 \% \mathrm{CO}_{2}$ in air (Fig. 1B). After the removal of cumulus cells, oocytes extruding the first polar body (PB) were considered to be matured (Fig. 1C). The accuracy of this criterion for nuclear maturation was $91.7 \%(22 / 24)$ when some of the PB-extruding oocytes were subjected to whole-mount preparation (Fig. 1D).

\section{Statistical analysis}

Percentage data in each replicate were analysed by one-way ANOVA after arcsin transformation. Means were compared by Fisher's least significant difference test (PLSD-test) using the StatView program (Abacus 

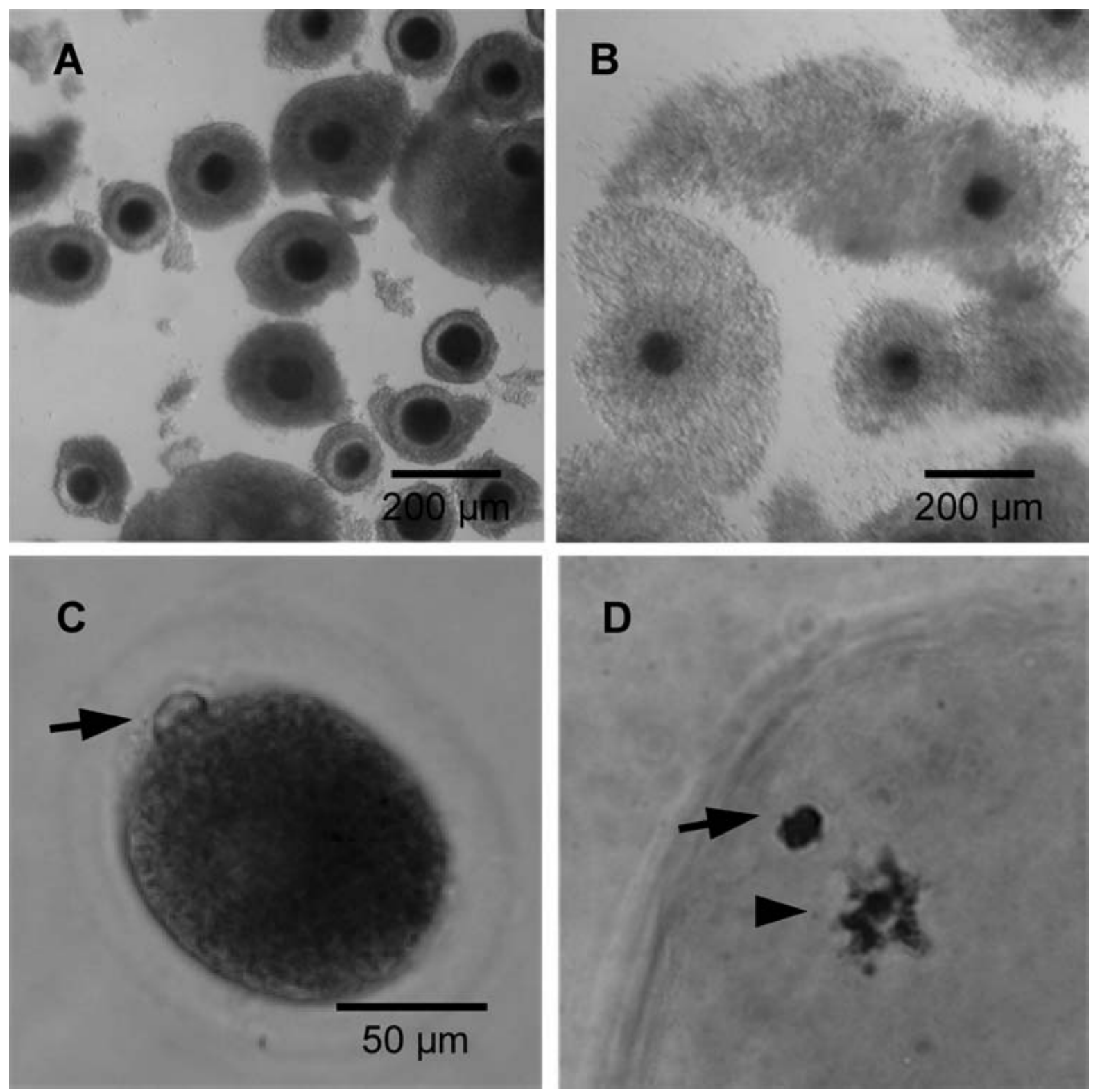

Figure $1(A)$ Minke whale COCs used for vitrification. Evenly granulated oocytes were enclosed with compact cumulus cell layers. (B) The COCs after warming and IVM. Cumulus cell layers were expanded. (C) An oocyte extruding the first polar body (arrow). (D) Whole-mount preparation of a polar body-extruding oocyte (arrow, first polar body; arrowhead, second metaphase plate).

Concepts, Berkeley, CA). A value of $p<0.05$ was chosen as an indication of significance.

\section{Results}

The mean recovery rate per prepubertal ovary was higher than that per adult ovary ( 21.0 vs 14.0 ; Table 1$)$. Regardless of the sexual maturity of the oocyte donors, approximately $40 \%$ of the total recovered COCs had compact cumulus layers and evenly granulated cytoplasm (Fig. 1A), and these were used for the experiments. Oocytes with compact cumulus layers and semicircular cytoplasm, classified as degenerated, were discarded, as were oocytes with expanded cumulus layers and denuded oocytes.

The effects of the cryodevice type and the donors' sexual maturity on the morphology of post-warm oocytes and the subsequent IVM are shown in Table 2. The proportion of morphologically normal COCs postwarming was significantly $(p<0.05)$ higher when the COCs were cryopreserved by Cryotop (adult origin, $88.4 \%$; prepubertal origin, $80.8 \%$ ) compared with the OPS (adult origin, $67.7 \%$; prepubertal origin, $64.2 \%$ ). 
Table 1 Morphological classification of COCs recovered from minke whales with different sexual maturity

\begin{tabular}{lccccr}
\hline & \multicolumn{5}{c}{ No. $(\%)$ of COCs recovered } \\
\cline { 2 - 6 } Sexual maturity of donors & Compact & Expanded & Denuded & Degenerated & Total \\
\hline Adult $(n=112)$ & 651 & 305 & 517 & 95 & 1568 \\
Prepubertal $(n=64)$ & $(41.5)$ & $(19.5)$ & $(33.0)$ & $(6.1)$ & $(100)$ \\
& 514 & 66 & 604 & 157 & 1341 \\
& $(38.3)$ & $(4.9)$ & $(45.0)$ & $(11.7)$ & $(100)$ \\
\hline
\end{tabular}

$n$, number of ovaries.

Table 2 Effects of cryodevice type and donors' sexual maturity on the morphology of post-warming oocytes and the subsequent IVM in minke whales

\begin{tabular}{lccccc}
\hline & & \multicolumn{4}{c}{ No. (\%) of oocytes } \\
\cline { 3 - 6 } Cryodevice & Donor's sexual maturity & Vitrified-warmed & Normal morphology & Cultured & Extruding first PB \\
\hline Cryotop & Adult & 198 & $175(88.4)^{a}$ & 175 & $51(29.1)^{a}$ \\
& Prepubertal & 172 & $139(80.8)^{a}$ & 139 & $20(14.4)^{b}$ \\
OPS & Adult & 217 & $147(67.7)^{b}$ & 147 & $21(14.3)^{b}$ \\
& Prepubertal & 162 & $104(64.2)^{b}$ & 104 & $11(10.6)^{b}$ \\
\hline
\end{tabular}

$\mathrm{PB}$, polar body.

${ }^{a, b}$ Different superscripts within columns are significantly different ( $p<0.05 ; 7$ replicates).

The oocyte maturation rate, as assessed by first PB extrusion, was significantly $(p<0.05)$ higher in the adult/Cryotop group (29.1\%) compared with the prepubertal/Cryotop group $(14.4 \%)$, the adult/OPS group (14.3\%) and the prepubertal/OPS group (10.6\%).

\section{Discussion}

In our previous study (Asada et al., 2000), conventional freezing of GV-stage minke whale oocytes was not fully successful, as the proportions of post-thaw oocytes that recovered with normal morphology and of those that matured in vitro were only $39.3-40.0 \%$ and 0 $3.3 \%$, respectively. However, in the present study, the survivability of GV-stage minke whale oocytes was improved by applying minimum volume cooling methods, such as the Cryotop and OPS methods. The Cryotop and OPS methods have been used for oocytes and/or embryos in cattle (Vajta et al., 1998; Tominaga \& Hamada, 2001), pigs (Esaki et al., 2004), rabbits (Hochi et al., 2004) and humans (Kuwayama \& Kato, 2000). The proportion of post-warm oocytes with normal morphology was significantly higher when the Cryotop rather than the OPS was used as the cryodevice, and a maximum proportion of post-warm oocytes extruding the first PB after IVM of $29.1 \%$ was obtained. As far as the cooling rate is concerned, which may influence the survivability post-vitrification, the Cryotop $\left(-23000{ }^{\circ} \mathrm{C} / \mathrm{min}\right)$ provides a higher rate than the OPS $\left(-16700^{\circ} \mathrm{C} / \mathrm{min}\right)$.
The developmental capacity of in vitro matured oocytes from prepubertal animals is in general lower than that from adult animals, as shown by Revel et al. (1995), who reported that the cytoplasmic maturation of calf oocytes was abnormal. Differences between prepubertal and adult oocytes have been characterized by different patterns in neosynthetic proteins (Khatir et al., 1998) and energy metabolism (Gandolfi et al., 1998), cytoplasmic organelle migration (Damiani et al., 1996) and reduced activity of some enzymes (Driancourt et al., 2001). Thus, a lower capacity to complete meiosis in oocytes derived from prepubertal whales may be supported by these findings.

All the adult oocyte donors used in the present study were found to be pregnant at various stages of gestation. That the oocyte donors were pregnant whales may have led to the limited IVM rate $(<30 \%)$ in the present study. Torner et al. (2003) reported that the time course of nuclear configuration during IVM in oocytes derived from pregnant camels is different from that in oocytes derived from non-pregnant camels. The earlier onset of apoptosis in COCs derived from non-pregnant donors possibly determines the faster progression of the oocytes through the final stages of meiosis (Torner et al., 2003; Alm et al., 2000). A direct comparison of the oocyte maturation capacity between pregnant and non-pregnant cows indicated that the IVM rate for oocytes from pregnant cows $(89.9 \%)$ was lower than that for oocytes from non-pregnant cows (95.4\%) (Chohan \& Hunter, 2003). 
The previous IVM system for whale oocytes (Fukui et al., 1997a; Asada et al., 2001a) included approximately 310 mosmol M199 supplemented with $15-20 \%$ fetal whale serum (FWS) for the IVM medium and 96-120 h as the culture period, resulting in an approximately $30 \%$ IVM rate in fresh oocytes. The preliminary measurement of osmolarity for $\mathrm{wFF}$ led us to prepare a modified IVM medium in the present study. The modifications resulted in the production of matured oocytes after vitrification and warming at a 30\% IVM rate, with full expansion of cumulus cells (Fig. 1B), after IVM culture for $40 \mathrm{~h}$ instead of 96-120 h. Osmolarity of the IVM medium is among the important factors influencing the capacity for development to blastocysts after IVF. In pigs, development of porcine oocytes is poor when the osmolarity of the medium is unsuitable (Funahashi et al., 1994, 1996; Yamauchi et al., 1999). The higher osmolarity in wFF $(397.9 \pm 3.1$ mosmol), compared with that of bovine follicular fluid (data not shown), may be one of characteristics specific to marine mammals. Further improvements are required for the IVM system for whale oocytes.

The criterion for oocyte survival used in the present study was the extrusion of the first PB, because the post-warming oocytes were forwarded to examine further developmental potential by ICSI. The overall proportion of ICSI oocytes exhibiting two or more cell nuclei after in vitro culture was $14.8 \%(12 / 81)$; the details of ICSI were not described in this paper. The poor developmental potential after ICSI may be due to the unestablished culture system for presumptive zygotes and possibly to insufficient stimuli for oocyte activation after ICSI.

In conclusion, GV-stage oocytes from adult minke whales can survive vitrification with the Cryotop as the cryodevice, and the present results show that the Cryotop is superior to the OPS for vitrification of minke whale immature oocytes.

\section{Acknowledgements}

The authors thank the captain and crew members of the research base ship Nisshin-maru for their help in obtaining minke whale ovaries.

\section{References}

Alm, H., Torner, H., Heleil, B., Srsen, V. \& Kuzmina, T. (2000). Apoptosis and pyknosis in cumulus cells from bovine cumulus-oocyte complexes before and after maturation in vitro (abstract). Theriogenology 53, 447.

Asada, M., Horii, M., Mogoe, T., Fukui, Y., Ishikawa, H. \& Ohsumi, S. (2000). In vitro maturation and ultrastructural observation of cryopreserved minke whale (Balaenoptera acutorostrata) follicular oocytes. Biol. Reprod. 62, 253-9.
Asada, M., Tetsuka, M., Ishikawa, H., Ohsumi, S. \& Fukui, Y. (2001a). Improvement on in vitro maturation, fertilization and development of minke whale (Balaenoptera acutorostrata) oocytes. Theriogenology 56, 52133.

Asada, M., Wei, H., Nagayama, R., Tetsuka, M., Ishikawa, H., Ohsumi, S. \& Fukui, Y. (2001b). An attempt at intracytoplasmic sperm injection of frozen-thawed minke whale (Balaenoptera bonaerensis) oocytes. Zygote 9, 299307.

Chohan, K.R. \& Hunter, A.G. (2003). Effect of reproduction status on in vitro developmental competence of bovine oocytes. J. Vet. Sci. 4, 67-72.

Damiani, P., Fissore, R.A., Cibelli, J.B., Long, C.R., Balise, J.J., Robl, J.M. \& Duby, R.T. (1996). Evaluation of developmental competence, nuclear and ooplasmic maturation of calf oocytes. Mol. Reprod. Dev. 45, 521-34.

Driancourt, M.A., Reynaud, K. \& Smitz, J. (2001). Defferences in follicular function of 3-month-old calves and mature cows. Reproduction 121, 463-74.

Esaki, R., Ueda, H., Kurome, M., Hirakawa, K., Tomii, R., Yoshioka, H., Ushijima, H., Kuwayama, M. \& Nagashima, H. (2004). Cryopreservation of porcine embryos derived from in vitro matured oocytes. Biol. Reprod. 71, 432-7.

Fukui, Y., Mogoe, T., Ishikawa, H. \& Ohsumi, S. (1997a). Factors affecting in vitro maturation of minke whale (Balaenoptera acutorostrata) follicular oocytes. Biol. Reprod. $56,523-8$.

Fukui, Y., Mogoe, T., Ishikawa, H. \& Ohsumi, S. (1997b). In vitro fertilization of in vitro matured minke whale (Balaenoptera acutorostrata) follicular oocytes. Mar. Mamm. Sci. 13, 395-404.

Funahashi, H., Cantley, T.C., Stumpf, T.T., Terlouw, S.L. \& Day, B.N. (1994). Use of low salt culture medium for maturation of porcine oocytes is associated with elevated oocyte glutathione levels and enhanced pronuclear formation after in vitro fertilization. Biol. Reprod. 51, 6339.

Funahashi, H., Kim, N.H., Stumpf, T.T., Cantley, T.C. \& Day, B.N. (1996). Presence of organic osmolytes in maturation medium enhances cytoplasmic maturation of porcine oocytes. Biol. Reprod. 54, 1412-19.

Gandolfi, F., Milamesi, E., Pocar, P., Luciano, A.M., Brevini, T.A.L. \& Acocclla, F. (1998). Comparative analysis of calf and cow oocytes during in vitro maturation. Mol. Reprod. Dev. 49, 168-75.

Hochi, S., Akiyama, M., Minagawa, G., Kimura, K. \& Hanada, A. (2001). Effect of cooling and warming rates during vitrification on fertilization of in vitro-matured bovine oocytes. Cryobiology 42, 69-73.

Hochi, S., Terao, T., Kamei, M., Kato, M., Hirabayashi, M. \& Hirao, M. (2004). Successful vitrification of pronuclearstage rabbit zygotes by minimum volume cooling procedure. Theriogenology 61, 267-75.

Khatir, H., Lonergan, P. \& Mermillod, P. (1998). Kinetics of nuclear maturation and protein profiles of oocytes from prepubertal and adult cattle during in vitro maturation. Theriogenology 50, 917-29.

Kong, I.K., Lee, S.I., Cho, S.G., Cho, S.K. \& Park, C.S. (2000). Comparison of open pulled straw (OPS) vs glass 
micropipette (GMP) vitrification in mouse blastocysts. Theriogenology 53, 1817-26.

Kuwayama, M. \& Kato, O. (2000). All-round vitrification method for human oocytes and embryos (abstract). J. Assist. Reprod. Genet. 17, 477.

Lane, M., Bavister, B.D., Lyons, E.A. \& Forest, K.T. (1999). Containerless vitrification of mammalian oocytes and embryos. Nat. Biotechnol. 17, 1234-6.

Martino, A., Songsasen, N. \& Leibo, S.P. (1996). Development into blastcyst of bovine oocytes cryopreserved by ultrarapid cooling. Biol. Reprod. 54, 1059-69.

Revel, F., Mermillod, P., Peynot. N., Renard, J.P. \& Heyman, Y. (1995). Low developmental capacity of in vitro matured and fertilized oocytes from calves compared with that of cows. J. Reprod. Fertil. 103, 11520.
Tominaga, K. \& Hamada, Y. (2001). Gel-loading tip as container for vitrification of in vitro-produced bovine embryos. J. Reprod. Dev. 47, 267-73.

Torner, H., Heleil, B., Alm, H., Ghoneim, I.M., Srsen, V., Kanitz,W., Tuchscherer, A. \& Fattouh, E.M. (2003). Changes in cumulus-oocyte complexes of pregnant and nonpregnant camels (Camelus dromedaries) during maturation in vitro. Theriogenology 60, 977-87.

Vajta, G., Holm, P., Kuwayama, M., Booth, PJ., Jacobsen, H., Greve, T. \& Callesen, H. (1998). Open pulled straw (OPS) vitrification: a new way to reduce cryoinjuries of bovine ova and embryos. Mol. Reprod. Dev. 38, 290-300.

Yamauchi, N., Sasada, H., Soloy, E., Dominko, T., Kikuchi, K. \& Nagai, T. (1999). Effects of hormones and osmolarity in the culture medium on germinal vesicle breakdown of porcine oocytes. Theriogenology 52, 153-62. 